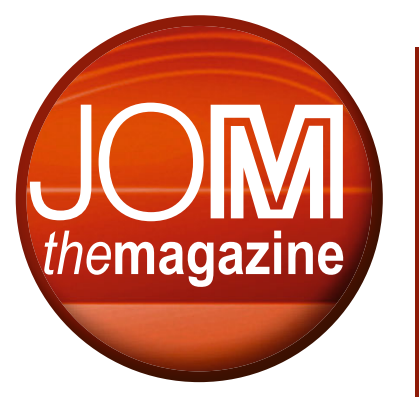

Manuel Abad

Julio Aguilar

Sreekanth Akarapu

Osman Anderoglu

Martin Anselm

Beth Armstrong

David Armstrong

Raul Arrabal

Singanahally Aruna

Olufemi Babalola

Ingo Bardenhagen

Burak Basaran

Ben Beake

Allison Beese

Hongbin Bei

Sanjit Bhowmick

Elena Boccardi

Herbert Boeckels

Raj Bordia

Nicola Bosso

Patrick Bowen

Geoff Brennecka

Tom Broderick

David Browne

Clifton Bumgardner

A. A. Burbelko
Carlos Capdevila
John Carpenter
Ellen Cerreta

Samrat Chaudhary

Nikhilesh Chawla

Chris Chen

Hai-Lin Chen

\section{Thanks to Our 2015 Peer Reviewers}

JOM extends a sincere thank-you to the reviewers who helped to ensure that the papers published in $\mathbf{2 0 1 5}$ met the high quality standards its readers have come to expect. The journal's growth in recent years has meant an elevated, expanded role for peer reviewers. The prompt, thorough, and fair reviews by these volunteers is much appreciated.
Mengjun Chen

Ming Chen

Po-Yu Chen

Shanshan Chen

Yang-yuan Chen

Youxing Chen

Yuzhen Chen

Perry Cheung

Brian Chmielowiec

Kester Clarke

James Cole

Peter Collins

Stefano Corni

Steve Coryell

Eric Cotts

Dan Coughlin

Chunxiang Cui

Euel Cutshall

Sefa Dag

Saverio D'Agostino

Neal Dando

James Dankwah

Mark Davis

Emmanuel De Moor

Aritra Dhar

Kevin Ding

Xuli Ding

Vasileios Drakonakis

Sebastien Dryepondt

Kui Du

Sergei Dudarev

Marc Dupuis

Thomas Eagar
James Earthman

Donna Ebenstein

Danny Edwards

Timothy Eisele

Ceren Erust

Juan Escobedo

Mohsen Eshraghi

Massimiliano Fabbricino

Adam Farrow

Sergio Felicelli

Kip Findley

Steven Fitzgerald

John Foltz

Elizabeth Friis

Tim Furnish

Amber Genau

Reza Ghaffarian

John Gibbs

Paul Gibbs

Michael Gibson

Gultekin Goller

Sarma Gorti

John Grandfield

Yijia Gu

Zhiyong Gu

Xiaofei Guan

E. Gegum Gulsoy

Manoj Gupta

Neha Gupta

Robert Hackenberg

Micah Hackett

Bengt Hallstedt

Bing Han
Carol Handwerker

Aman Haque

Khalid Hattar

Peter Hayes

James Haynes

Mingsheng $\mathrm{He}$

Sayyed Mahdi Hejazi

Qian Huang

Xiaodi Huang

Jiann-Yang Hwang

Margaret Hyland

Shadia Ikhmayies

Seth Imhoff

Saumyadeep Jana

Vikram Jayaram

Xiujuan Jiang

Rhys Jones

Kang Jun

In Hwa Jung

Farhoud Kabirian

Joshua Kacher

Kumar Kandasamy

Singon Kang

Monica Kapoor

Shyamprasad Karagadde

Ursula Kattner

Brett Kaufman

Matthew Kerr

Rohit Khanna

Daniel Kiener

Hyoung Kim

Keun-Soo Kim

Jamie Kimberley 


\begin{tabular}{|c|c|c|c|}
\hline Seda Kizilel & Prabhakar Mohan & Mohammad Mahdi Salehi & Guofeng Wang \\
\hline Steinar Kolås & Yousef Mohassab & Hossein Sepehri-Amin & Jian Wang \\
\hline Eswara Korimilli & Maryam Mohri & Paul Shade & Kegang Wang \\
\hline M. Kramer & Loris Molent & Zhiwei Shan & Mingyu Wang \\
\hline Jamie Kruzic & Sergio Monteiro & Shuai Shao & Xiangwen Wang \\
\hline A. GuruSampath Kumar & Benjamin Morrow & Yao Shen & Xinnan Wang \\
\hline Abhishek Kumar & Stefano Morsut & Rongpei Shi & Yanli Wang \\
\hline Nilesh Kumar & Takashi Nagai & Wenwu Shi & Yinmin (Morris) Wang \\
\hline Sandeep Kumar & Ha-Duong Ngo & Yasushi Shibuta & Yuxing Wang \\
\hline Debrupa Lahiri & Nikan Noorbehesht & Tolou Shokuhfar & Zhao-Xi Wang \\
\hline Jonghyun Lee & Michael Notis & Qi Feng Shu & Juri Wehrs \\
\hline Simon Lekakh & Václav Ocelík & Amit Shyam & Chao-Chen Wei \\
\hline Laura Lewis & Elizabeth Opila & John Slotwinski & Christine Wenzl \\
\hline Lan Li & Dmytro Orlov & Malcolm Snead & Jeffrey Wheeler \\
\hline Qian Li & Mrutyunjay Panigrahi & Polina Snugovsky & Robert Wheeler \\
\hline Shoupeng Li & Baroch Pavel & Hong Yong Sohn & Mike Widom \\
\hline Xiaodong Li & Erik Pavlina & Kemal Sonmez & Dane Wilson \\
\hline Yangang Liang & Mathew Peet & Jeff Spangenberger & Thomas G. Woodcock \\
\hline Joseph Licavoli & Zhiwei Peng & Marcel Spekowius & Zhenggang Wu \\
\hline Fengchao Liu & Fei Peng & Aaron Stebner & Kelvin Xie \\
\hline Jia Liu & John Perepezko & Erich Stergar & Baoxing Xu \\
\hline Lin Liu & Bruce Pint & Ziqi Sun & Peng Xu \\
\hline Shuhong Liu & Vinko Potocnik & Desikan Sundararajan & Clarissa Yablinsky \\
\hline Yong Liu & Adam Powell & Sami Syammach & Kenta Yamanaka \\
\hline Yue Liu & Greg Power & Alton Tabereaux & Fuqian Yang \\
\hline Terry Lowe & Radhakrishna Prabhu & Sesh Tamirisakandala & Huayi Yin \\
\hline Xiao-Gang Lu & Saurabh Puri & Zhi Tang & Lang Yuan \\
\hline A. Ludwig & Jonathan Puthoff & Aidong Tang & Onuralp Yücel \\
\hline Erik Luther & Junwei Qiao & Gordon Tatlock & Jing Zhan \\
\hline Jonathan Madison & Nima Rahbar & Mark Taylor & Chuan Zhang \\
\hline Rabindra Mahapatra & Jagannathan Rajagopalan & Toshiharu Teranishi & Haijun Zhang \\
\hline Mahmood Mamivand & Raul Rebak & Justin Tokash & Haitao Zhang \\
\hline Vaso Manojlovic & Barbara Rinderer & Vikas Tomar & Hongwen Zhang \\
\hline Guilhem Martin & Mario Rodriguez & L.D. Timmie Topoleski & Juhua Zhang \\
\hline Simon Middleburgh & Shari Rosenbloom & Damien Tourret & Lifeng Zhang \\
\hline \multirow{2}{*}{$\begin{array}{l}\text { Leonardo Fagundes } \\
\text { Rosemback Miranda }\end{array}$} & Marco Rossi & Piyush Upadhyay & Yifei Zhang \\
\hline & Franz Roters & Oswald Uwakweh & Yong Zhu \\
\hline Rajiv Mishra & Lee Rothleutner & Tomás Vargas & Silviya Zustiak \\
\hline Christian Mitterer & Ritesh Sachan & Cora Vasilescu & \\
\hline Seiji Miyashita & Akimasa Sakuma & Samuel Wagstaff & \\
\hline
\end{tabular}

